\title{
Clinical treatment for symptoms associated with temporomandibular disorder
}

\author{
Su-Gwan Kim, D.D.S., Ph.D. \\ Section Editor of JKAOMS \\ Department of Oral and Maxillofacial Surgery, School of Dentistry, Chosun University, Gwangju, Korea
}

Temporomandibular disorder (TMD) is a dysfunction of the temporomandibular joint (TMJ) and/or the masticatory musculature, both of which connect the mandible to the skull ${ }^{1}$. Generally, patients with TMD present with pain symptoms (e.g., jaw ache, earache, toothache, facial pain, and headache). Despite of various studies, the pathophysiological etiology of TMD remains unclear; however, these studies have led to improvements in diagnosis and treatment of TMD.

TMD is generally classified as a disorder of the either the TMJ or the musculature ${ }^{2}$. Disorders of the TMJ result from biomechanical imbalance between the disc and condyle ${ }^{2}$; this imbalance may induce disc interference disorders or internal derangements, which can be asymptomatic or symptomatic, and are triggered by inflammation in TMJ. Common disorders of the TMJ (e.g., disc displacement with/without reduction, retrodiscitis, TMJ subluxation, and osteoarthritic changes) may be accompanied by one or more signs (e.g., a painful or non-painful click, painful limitation of opening, orofacial pain, and progressive degeneration of TMJ articular (artilage) $)^{2}$.

Muscular disorder TMD is generally classified as myalgia, myofascial pain, and myositis. Myalgia may induce acute pain along with a dull ache as that caused by muscle injury or strain $^{2}$; sometimes the pain of muscle tension can be chronic. Myofascial pain may also manifest as a dull ache with varying intensity. In cases of myofascial pain, palpation of trigger points should duplicate the pain threshold ${ }^{3}$; therefore, clinicians can easily detect the myofascial pain trigger points and confirm the diagnosis. Myositis consists of localized transient muscle and facial tissue swelling caused by infection or injury ${ }^{4}$; mandibular movement and localized tenderness have a tendency to increase the pain threshold.

For patients with TMD, the aim of clinical management is to relieve the symptoms (pain with mandibular function, facial pain, headache, etc.) by restoring normal mandibular range of motion and normal masticatory and jaw function ${ }^{2}$. TMD clinical management can take the form of self-care programs, non-surgical medical care, and surgical care.

1) Self-care programs

Patient education for managing TMD symptoms should initially be performed frequently ${ }^{2}$; after receiving education on self-management of TMD symptoms patients report an immediate reduction in pain. Therefore, outcomes of clinical treatment might be alleviated by informing and reassuring the patient regarding their condition and symptoms.

2) Non-surgical medical care

Non-surgical medical care (e.g., physical therapy, pharmacotherapy, occlusal appliance therapy, and occlusal adjustment) is used to relieve symptoms in patients with TMD. Physical therapy using moist heat or cold compresses, or alternation of both modalities, have been reported to improve movement and relaxation of the jaw through restoring normal functions of the TMJ, mastication muscles, and cervical neck muscles ${ }^{4,5}$. Pharmacotherapy, one of the most effective forms of clinical management for TMD symptoms, usually involves analgesics, nonsteroidal anti-inflammatory drugs (e.g., ibuprofen, naproxen, and celecoxib), local anesthetics (procaine and lidocaine), oral and injectable corticosteroids for managing the TMJ pain, sodium hyaluronate injection to manage TMJ osteoarthritis, muscle relaxants (cyclobenzaprine, etc.), and antidepressants to manage myofascial pain ${ }^{2}$. Although occlusal appliance therapy can reduce joint and muscle pain, there is an associated risk of potential side effects ${ }^{6}$. Furthermore, there is insufficient evidence regarding the efficacy of occlusal adjustment for managing or preventing $\mathrm{TMD}^{7}$.

3) Surgical care

When non-surgical therapies are ineffective, surgical care is recommended for patients with symptomatic $\mathrm{TMD}^{8}$. Arthrocentesis is a conservative treatment that involves intraar- 
ticular lavage with or without deposition of corticosteroids, and is useful in cases with intraarticular restriction of movement ${ }^{9}$. Arthroscopy is a closed surgical procedure that allows direct internal observation of the $\mathrm{TMJ}^{10}$ and is useful in cases of TMJ hypomobility as well as in TMJ tissue sampling. Arthroscopy is an open surgical procedure used for reconstructing or replacing the TMJ in cases of neoplasia, bony or fibrous ankylosis, severe chronic arthritis, and severe chronic dislocations ${ }^{4}$. Oral and maxillofacial surgeons with expertise in TMJ surgery must carefully plan clinical treatment based on a variety of factors (e.g., chief complaint, degree of internal derangement, medical history, presenting symptoms, and diagnosis).

A number of recent studies regarding the pathophysiological etiologies or clinical management of TMD have provided the opportunity for greater standardization of TMD classification. These studies have also allowed for improvements in diagnosis and higher success rates for therapy. Currently, clinicians can provide correct diagnosis and clinical management of TMD based on a great deal of clinical experience and knowledge.

\section{Conflict of Interest}

No potential conflict of interest relevant to this article was reported.

\section{References}

1. Murphy MK, MacBarb RF, Wong ME, Athanasiou KA. Temporomandibular disorders: a review of etiology, clinical management, and tissue engineering strategies. Int J Oral Maxillofac Implants 2013;28:e393-414.

2. Romero-Reyes M, Uyanik JM. Orofacial pain management: current perspectives. J Pain Res 2014;7:99-115.

3. Simons DG, Travel JG, Simons LS. Myofacial pain and dysfunction: the trigger point manual. 2nd ed. Atlanta, GA: Lippincott Williams \& Wilkins; 1999.

4. de Leeuw R. Temporomandibular disorders. In: de Leeuw R, ed. Orofacial pain guidelines for assessment, diagnosis, and management. 4th ed. Chicago: Quintessence Publishing; 2008:158-76.

5. Danzig WN, Van Dyke AR. Physical therapy as an adjunct to temporomandibular joint therapy. J Prosthet Dent 1983; 49:96-9.

6. Klasser GD, Greene CS. Oral appliances in the management of temporomandibular disorders. Oral Surg Oral Med Oral Pathol Oral Radiol Endod 2009;107:212-23.

7. Koh H, Robinson PG. Occlusal adjustment for treating and preventing temporomandibular joint disorders. Cochrane Database Syst Rev 2003;(1):CD003812.

8. Helfrick JF. Parameters of care: a structured method for improving care. Tex Dent J 1994;111:7-8.

9. Nitzan DW, Dolwick MF, Martinez GA. Temporomandibular joint arthrocentesis: a simplified treatment for severe, limited mouth opening. J Oral Maxillofac Surg 1991;49:1163-7.

10. Buckley MJ, Merrill RG, Braun TW. Surgical management of internal derangement of the temporomandibular joint. J Oral Maxillofac Surg 1993;51(1 Suppl 1):20-7. 